\title{
AUTOPERCEPCIÓN DE LA ESCLEROSIS MÚLTIPLE: LA PERSONA FRENTE A SUS OCUPACIONES
}

\author{
SELF-PERCEPTION OF MULTIPLE SCLEROSIS: THE PERSON FACING THEIR \\ OCCUPATIONS
}

\section{Pablo Abal Rey', Nereida María Canosa Domínguez², Thais Pousada ${ }^{3}$}

\begin{abstract}
RESUMEN
Objetivos. La presencia de un diagnóstico de esclerosis múltiple (EM) compromete el desempeño ocupacional de las personas afectadas. Este estudio tuvo como objetivos conocer cómo la población con EM percibe su estado de salud y calidad de vida en relación a su cotidianidad, además de indagar en la perspectiva de los terapeutas ocupacionales acerca de la EM y las personas que la padecen.

Método. Se propuso una metodología mixta, empleando cualitativamente un análisis temático a través de entrevistas, grupo focal y la copm. Cuantitativamente, con un enfoque descriptivo se realizó un análisis estadístico de los datos tras administrar la LHS, SF-36 y cuestionario online para profesionales. Fueron 70 los participantes: 15 personas con EM y 55 terapeutas ocupacionales.

Resultados. Destacan las actividades de la vida diaria básicas (AVDB), instrumentales (AVDI) y la pérdida del rol de trabajador/a como principales limitaciones en el desempeño. Además, manifiestan que las necesidades de dicha población no se encuentran cubiertas desde el sistema público de salud, afectando esto a su calidad de vida. Los terapeutas ocupacionales expresan la necesidad de promover la investigación en este ámbito, destacando la intervención terapéutica en el medio natural del usuario.

Conclusiones. Las AVDB y AVDI son identificadas como un desafío en la cotidianidad de la población con EM, donde el rol de trabajador aporta gran significado a la identidad de la persona. Existe una necesidad cambio en el enfoque de la atención a las personas con EM, contemplando factores centrales para la cotidianeidad desde un prisma biopsicosocial.
\end{abstract}

\section{PALABRAS CLAVE}

Calidad de vida. Esclerosis Múltiple. Rehabilitación Neurológica. Terapia Ocupacional.

1 Terapeuta Ocupacional en SINAPSE Neurología. Coordinador de Investigación en el Grupo Mbody. Vocal de Ciencias de la Salud en la Agencia de Calidad para el Sistema Universitario de Galicia (ACSUG).Grado en Terapia Ocupacional (Universidad de A Coruña). Máster Universitario en Neurorrehabilitación (Institut Guttmann- Universitat Autònoma de Barcelona). https://orcid.org/o000-0003-1223-064X Calle Ourense, 14, 1º CP: 36630 - Cambados (Pontevedra).España.+34 627074805 / pablo.abal.rey@gmail.com

2 Terapeuta Ocupacional. Vicedecana de la Facultad de Ciencias de la Salud de la Universidad de A Coruña. Profesora y coordinadora del Grado en Terapia Ocupacional en la Universidad de A Coruña. Centro de Investigación en Tecnologías de la Información y de las Comunicaciones (CITIC). Grupo de Investigación de Tecnología Aplicada a la Investigación en Ocupación, Igualdad y Salud (TALIONIS).Diplomatura en Terapia Ocupacional (Universidad de A Coruña). Máster en Planificación y Dirección de Servicios Sociales (Universidad de A Coruña). Graduada Superior en Gerontología. Especialidad en Gerontología Social (Universidad de Santiago de Compostela). Máster en Asistencia e Investigación Sanitaria. Especialidad en Reeducación Funcional, Autonomía Personal y Calidad de Vida (Universidad de A Coruña).+34 669088487 / https://orcid.org/0000-0001-8527-9845 nereida.canosa@udc.es

3 Terapeuta Ocupacional. Enfermera. Profesora del Grado en Terapia Ocupacional en la Universidad de A Coruña. Doctora en Ciencias de la Salud. Centro de Investigación en Tecnologías de la Información y de las Comunicaciones (CITIC). Grupo de Investigación de Tecnología Aplicada a la Investigación en Ocupación, Igualdad y Salud (TALIONIS). Grado en Terapia Ocupacional (Universidad de A Coruña). Grado en Enfermería (Universidad de A Coruña). Máster en Asistencia e Investigación Sanitaria. Especialidad en Reeducación Funcional, Autonomía Personal y Calidad de Vida (Universidad de A Coruña). http://orcid.org/0000-0003-3356-0429. thais.pousada.garcia@udc.es.+34651 196913. 


\section{ABSTRACT}

Goal: The presence of a multiple sclerosis (MS) diagnosis, compromises the occupational performance of affected people. The purpose of the study was to find out how population which presents MS perceives their health status and quality of life, in relation to their daily lives. In addition, to investigate the perspective of occupational therapists about MS and people who suffer from it.

Methodology: A mixed methodology was proposed, using qualitative thematic analysis through interviews, focus group and the copM. Quantitatively, with a descriptive approach, a statistical data analysis was carried out after administering the LHS, SF-36 and online questionnaire for professionals. There were 70 participants: 15 people with MS and 55 occupational therapists.

Results: Activities of daily living ( $A D L$ ) and the loss of worker's role stand out as the main limitations in occupational performance. Furthermore, participants state that the needs of this population are not covered by the public health system. This affects their quality of life. Occupational therapists express the need for promoting research in this area, highlighting therapeutic intervention in the user's natural environment.

Conclusions: ADL and IADL are identified as a challenge in daily life of the MS community, where the worker's role brings a hug significance to person's identity. There is a need for a change in the approach to care of people with MS, considering central factors for everyday life from a biopsychosocial perspective.

\section{KEYWORDS}

Quality of life. Multiple Sclerosis. Neurological Rehabilitation. Occupational Therapy.

Recibido: 01/04/2020

Aceptado: 05/05/2021 


\section{INTRODUCCIÓN}

La Sociedad Española de Neurología (SEN), define la Esclerosis Múltiple (EM) como "una enfermedad crónica del sistema nervioso central (SNC) que se caracteriza por la existencia de inflamación, desmielinización, cicatrización glial y daño neuroaxonal, todo lo cual produce grados variables de lesión neurológica persistente" (García, Ara, Fernández, Landete, Moral, \& RodríguezAntigüedad, 2017).

A nivel mundial está descrito que la EM muestra una relación directa entre la prevalencia e incidencia de la enfermedad y una distribución geográfica concreta planteada por Kurtzke en 1975 (Kurtzke, 2009), caracterizada por un gradiente latitudinal, aumentando la prevalencia a medida que se aleja de la línea ecuatorial (García-Cano \& García-Cano, 2017; Koch-Henriksen \& Sørensen, 2010).

En cuanto al alcance o epidemiología de la enfermedad, Esclerosis Múltiple España (EME) aporta las siguientes cifras que provienen de la SEN en el año 2019:

- 2.500.00o personas padecen EM a nivel mundial.

- 700.000 personas tienen EM en Europa.

- 55.00o personas poseen un diagnóstico de EM en España (Esclerosis Múltiple España, 2019).

Por tanto, la tasa de prevalencia en España se considera media-alta, donde las cifras más elevadas de la misma tienen origen en zonas con latitudes más altas. A lo largo de las últimas décadas se ha producido un aumento de esta prevalencia de manera paulatina, situándose la cifra, actualmente, entre los $80-180$ casos por cada 100.000 habitantes (Pérez-Carmona, Fernández-Jover \& Sempere, 2019)

La Federación Gallega de Esclerosis Múltiple (FEGADEM) estima que en la comunidad autónoma de Galicia son alrededor de 3.00o las personas registradas, mostrando probablemente la mayor incidencia de EM de toda España. Actualmente, la cifra se sitúa en unos 123 afectados por cada 100.00o habitantes (Federación Gallega de Esclerosis Múltiple, 2018).

Dado que actualmente la EM no cuenta con un tratamiento curativo, es imprescindible llevar a cabo estrategias encaminadas a prevenir la evolución y complicaciones de la enfermedad, así como el afrontamiento y aceptación del diagnóstico. Uno de los aspectos que se encuentran más olvidados en esta población, es el de la autopercepción del estigma relacionado con su cotidianeidad. La definición de estigma ha evolucionado notablemente. De la clásica concepción de estigma que Goffman proponía como un atributo o característica que expresa una identidad social devaluada, se ha llegado a ampliar el concepto proponiendo concebir el estigma como un proceso social en lugar de como un atributo. Este proceso de estigmatización comienza cuando los grupos dominantes de una sociedad identifican ciertas diferencias humanas, culminándose cuando dichas diferencias desencadenan en diversas formas de rechazo, exclusión y discriminación (Miric, Álvaro, González \& Torres, 2017).

Resulta de interés el establecer la íntima relación existente entre el concepto de estigma y el de auto-concepto. El término auto-concepto, al igual que el de estigma, también ha sido acto de modificaciones y evolución a lo largo de los años. Tradicionalmente fue entendido como el conjunto de percepciones que mantiene una persona sobre sí misma a partir de la valoración personal y de los otros significativos, avanzando en el siglo Xxı hasta el concepto que tiene de sí misma como un ser físico, social y espiritual (García \& Musitu, 2014). Kielhofner por su parte, reconoce que la participación en las actividades productivas desempeña un papel importante en la creación de la identidad del ser humano (Kielhofner, 2008).

Ambos conceptos muestran una gran influencia en la cotidianeidad de las personas con EM, dado que el procesamiento resultante de un cuerpo que reacciona, sumado al enfrentamiento que estos producen en los ambientes y a una actitud no siempre favorable (Kos, Duportail, Meirte, Meeus, D'hooghe, Nagels \& Nus, 2016), genera que estas personas muestren problemas en la participación de actividades cotidianas y por tanto obtengan un resultado negativo en el equilibrio ocupacional.

Además, el concepto de ocupación se encuentra fuertemente relacionado con la calidad de vida, dado que las personas poseen la capacidad para seleccionary ser partícipes de sus ocupaciones, "disfrutar de la vida ycontribuir en la sociedad"(Kielhofner, 2006).

La calidad de vida es un aspecto de relevancia al hacer referencia a la presencia de alguna enfermedad crónica. 
No existen tratamientos curativos, sino medidas terapéuticas enfocadas a detener el avance de la patología y a mantener las funciones preservadas (Cano, 2015). Actualmente, es abundante la literatura que aborda el concepto de calidad de vida como eje central de estudio (Yu \& Mathiowetz, 2014b; Yu \& Mathiowetz, 2014a). Con ello, permiten tanto valorar las condiciones derivadas de la enfermedad como conocer la percepción que la persona afectada tiene de su situación vital, especialmente en las áreas de bienestar físico, social y emocional (Arbinaga, 2003).

Por todo ello, se considera necesario y pertinente la realización de un estudio que, desde terapia ocupacional (TO), aúne los conceptos previamente expuestos y persiga cómo objetivo (1) conocer cómo las personas con EM perciben su estado de salud y calidad de vida con relación a su cotidianidad y (2) determinar la percepción de los profesionales de TO acerca de la EM y la percepción del usuario.

\section{MÉTOdo}

Para la elaboración de esta investigación, se ha considerado oportuno utilizar una metodología mixta (cuantitativa y cualitativa) siguiendo un diseño de triangulación concurrente. El conseguir una aproximación global al objeto del estudio, permite un aumento del conocimiento y ayuda a comprender la realidad del fenómeno que se estudia.

La investigación mixta se sustenta en las fortalezas de cada método y no en sus debilidades. Con el enfoque mixto se exploran distintos niveles del problema de estudio. Estos diseños logran obtener una mayor variedad de perspectivas del problema: frecuencia, amplitud y magnitud (cuantitativa), así como profundidad y complejidad (cualitativa), denominada como "riqueza interpretativa" (Hernández, Fernández \& Baptista, 2010).

Se ha seguido un diseño de investigación mixto específico: el diseño de triangulación concurrente. Este diseño es el más popular en la investigación, y se utiliza cuando el investigador pretende confirmar o corroborar resultados y efectuar una validación cruzada de los datos cualitativos y cuantitativos. De manera simultánea se recogen y analizan datos cuantitativos y cualitativos sobre el objeto de investigación (Hernández, Fernández \& Baptista, 2010).
En cuanto a la vertiente cualitativa, se empleó un análisis temático, característico de este tipo de investigación, con un enfoque fenomenológico y descriptivo, ya que su objetivo principal es la búsqueda de significados individuales y compartidos que las personas con EM ofrecen de su cotidianeidad y sus ocupaciones. Este análisis se basa en la descripción de narraciones basadas en su historia de vida, mediante un proceso inductivo, creando códigos y áreas temáticas, y llevando a cabo una continua revisión de los hallazgos (Vaismoradi, Turunen, \& Bondas, 2013).

Por otro lado, en lo relativo a la vertiente cuantitativa, se ha realizado un análisis estadístico de los datos cuantitativos optando por un estudio descriptivo y transversal, recogiendo datos reales sobre la calidad de vida, independencia y auto-concepto de la persona en su día a día.

El trabajo de campo se ha realizado en la Federación Gallega de Enfermedades Raras y Crónicas (FEGEREC) en A Coruña. FEGEREC está compuesta por un total de once asociaciones de enfermedades raras y crónicas, afectados de enfermedades ultra raras, familias y socios colaboradores. El objetivo de FEGEREC es mejorar la calidad de vida de las personas afectadas por una enfermedad rara y/o crónica y la de sus familias, además de defender sus derechos y ser su voz ante la sociedad, administraciones y entidades (Federación Gallega de Enfermedades Raras y Crónicas, 2018).

El período de estudio ha comprendido desde septiembre de 2018 hasta junio de 2019. Para el correcto desarrollo del estudio, se certifica que la investigación ha sido llevada a cabo respetando totalmente la normativa ética vigente en la Declaración de Helsinki, garantizando a todos los participantes del estudio, la confidencialidad y anonimato en referencia a sus datos e información personal. Además, el estudio fue presentado al Comité Ético de FEGEREC con la posterior aprobación de la entidad.

\section{Participantes}

Se ha empleado un muestreo no probabilístico e intencional. Además, también se ha establecido un muestreo teórico, dado que el número de participantes ha sido acotado en función de la saturación teórica. 
Los criterios de inclusión adoptados para los usuarios han sido: encontrarse asociados a FEGEREC, presentar un diagnóstico de EM, ser mayor de 18 años y pertenecer al área geográfica gallega. Por parte de los profesionales: poseer la diplomatura o grado en TO y encontrarse ligado al ámbito de actuación de TO en EM.

Los usuarios que presentasen un deterioro cognitivo y/o dificultades en la comunicación que impidiese realizar adecuadamente la entrevista y administración de las herramientas estandarizadas, así como aquellos que no acudiesen a ningún servicio de rehabilitación funcional fueron excluidos. Por otro lado, los profesionales con menos de 1 año de experiencia práctica en el ámbito de la EM tampoco participaron en el estudio.

La muestra seleccionada estuvo compuesta finalmente por 15 personas con un diagnóstico de EM, previo consentimiento voluntario. De los 15 participantes, 10 de ellos presentaban una Esclerosis Múltiple Remitente Recurrente (EMRR), 4 cursaban la forma Primaria Progresiva y 1 de ellos la Secundaria Progresiva.

La identidad de los quince participantes en el estudio de investigación fue codificada a través de la asignación de un código alfanumérico, con el objetivo de mantener la confidencialidad. La asignación ha sido elaborada en función del momento de participación en la entrevista inicial, iniciándose por el código U1 correspondiente a la inicial de la palabra "Usuario" seguida de números del uno al quince.

Además, por parte de los participantes profesionales, 55 respondieron al cuestionario. Sus identidades fueron también codificadas a través de un código alfanumérico, iniciándose por el código $\mathrm{P}_{1}$ correspondiente a la palabra "Profesional" seguida de números del 1 al 55.

\section{RECOGIDA DE DATOS}

Los métodos de recogida de datos escogidos han sido seis:

London Handicap Scale (LHS): Esta escala comprende 6 aspectos que se puntúan de 1 a 6: movilidad, independencia física, ocupación, integración social, orientación y autosuficiencia económica. La LHS destaca por su fácil administración, escasa variabilidad y rápida aplicación
(Harwood \& Ebrahim, 1995; Harwood et al., 1994; Prieto-González, 2000).

Cuestionario de salud SF36 (Health Survey SF-36): Este cuestionario deriva del Medical Outcomes Study (MOS-SF-36) y es una de las herramientas genéricas más empleadas para evaluar la calidad de vida en relación con el estado de salud. El SF-36 está conformado por dos secciones: 1) el propio cuestionario, que abarca 35 ítems que aportan información acerca de tres áreas: situación funcional, bienestar emocional y estado de salud general, y 2) una cuestión que hace referencia al cambio percibido en el estado de salud en el tiempo (Bermejo, Porta, Díaz \& Martínez, 2008).

Cuestionario online para profesionales: para conocer la perspectiva de los terapeutas ocupacionales que trabajan con población diagnosticada de EM, se ha administrado un cuestionario online que se envió a diferentes profesionales del país a través de diversas asociaciones y colegios oficiales del territorio español. El cuestionario ha estado abierto a respuestas durante los meses de febrero y marzo de 2019.

Dicho cuestionario, se ha desarrollado basándose en el cuestionario para pacientes presentado en el Informe "ME INTERESA" titulado: "Estudio sobre la Percepción de las personas con EM sobre su calidad de vida" (Novartis, 2018).

Medida Canadiense del Desempeño Ocupacional (COPM): se ha empleado dicha herramienta, propia de la disciplina de TO, con el objetivo de conocer e indagar en las prioridades ocupacionales de los participantes.

Entrevista semiestructurada individual: Para llevar a cabo la recogida de datos se empleó una entrevista semiestructurada (ES) cuyo guion fue específicamente elaborado para este trabajo, y que se aplicó a los participantes una vez que estos habían aprobado su conformidad para formar parte del estudio. En el momento previo a la realización de las ES, se les facilitó a cada uno la Hoja de información al/la participante, aportando un tiempo suficiente para que fuese leída, así como la Hoja de Consentimiento Informado. Todos los participantes dieron su consentimiento para la grabación en audio de la conversación de la ES y para que esta se transcribiese posteriormente. 
Grupo focal o de discusión: El grupo focal o de discusión, se ha llevado a cabo con la participación de diez de los quince informantes de las entrevistas, durante dos horas y media, empleando una grabadora como instrumento para la recogida de información. Este grupo focal se ha desarrolló en horario de mañana, en la misma sala establecida para las ES. En él, los usuarios que acudieron habían de traer y presentar una imagen o fotografía con la que se sintiesen identificados y comentar brevemente el motivo, para iniciar la sesión. Los temas planteados en el grupo se establecieron tras el análisis de la información obtenida en las ES individuales.

\section{Gestión y análisis de datos}

El método de análisis de datos utilizado en el estudio ha sido diferente, dadas las dos tendencias metodológicas empleadas.

En cuanto a la vertiente cualitativa, como ya se ha mencionado, se empleó un análisis temático con un enfoque fenomenológico y descriptivo. Este tipo de análisis ha permitido identificar, analizar e informar sobre patrones dentro del conjunto de datos.

En la metodología cuantitativa, las variables numéricas se expresan como media $(M)$, rango $(R)$ y desviación típica (SD), mientras que las variables categóricas se muestran con su frecuencia absoluta y porcentaje válido.

Además de la descripción de los datos y variables del estudio, se ha considerado realizar un análisis inferencial con el fin de determinar posibles relaciones significativas entre las variables, especialmente en las que se encontraban implicadas en el objetivo principal del estudio.

De manera previa, se realizó la prueba de KolmogorovSmirnov para comprobar que las variables no se comportan como distribución normal, por lo que se aplicaron las pruebas no paramétricas. La asociación entre variables numéricas se analizó con la prueba Rho de Spearman y la relación entre variables categóricas se ha analizado con la prueba Chi Cuadrado.

En lo referente a la asociación entre variables numéricas y categóricas, se realizó la comparación de medias con la prueba $U$ de Mann Whitney. Se estableció como nivel de significación para el contraste de hipótesis el 5\%.
Por último, se ha aplicado la prueba $\alpha$ de Cronbach para conocer la consistencia interna de la LHS y del Cuestionario de Salud SF-36.

El tratamiento de estos datos estadísticos se ha realizado a través de la versión 25 del programa SPSS.

\section{Resultados}

\section{Resultados cuantitativos}

\section{CARACTERÍSTICAS SOCIODEMOGRÁFICAS DE LA MUESTRA}

El estudio ha incluido a un total de 70 participantes: 15 usuarios $(U)$ y 55 profesionales $(\mathrm{P})$. Dentro del grupo de los usuarios, se incluyeron 5 hombres y 10 mujeres, con una media de edad de 44,9 años $(S D=11,5)$.

En el grupo de profesionales, el 89,1\% han sido mujeres y el 10,9\% restante hombres, con una edad media de 33 años $(S D=6,9)$

Los participantes profesionales, proceden de diversos puntos de la geografía española, obteniendo un mayor número de respuestas al cuestionario desde las comunidades autónomas de Castilla y León (25,5\%), Galicia $(23,6 \%)$ y Madrid (21,8\%). El 29,1\% restante se divide entre las demás comunidades autónomas españolas.

En la Figura 1 se expone la procedencia heterogénea del ámbito de trabajo de dichos profesionales, siendo el sector asociativo el que ha aportado mayor representación, con un $45,5 \%$. 
FIGURA 1. ÁMBITO DE PROCEDENCIA DE LOS PROFESIONALES

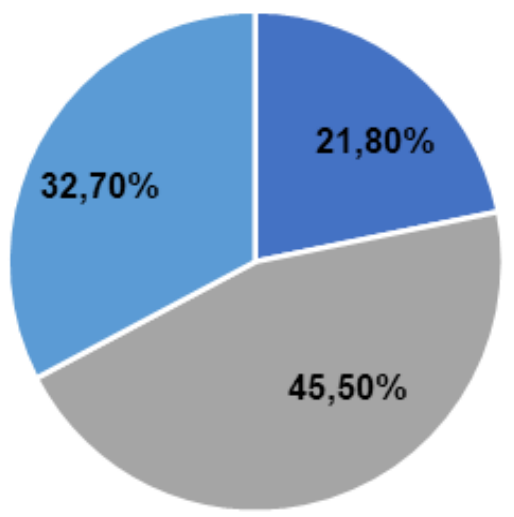

- Hospitalario "Asociativo -Privado

\section{ANÁLISIS DE LOS DATOS CUANTITATIVOS DE LAS PERSONAS AFECTADAS}

\section{London Handicap Scale}

Tras el análisis de los resultados obtenidos con la LHS, se determinó que el nivel medio de independencia es de $0,592(\mathrm{SD}=0,17)$, sobre una puntuación máxima de 1, siendo el de "ocupación" el ítem en el que se muestra una peor puntuación media, siendo de -0,022 $(\mathrm{SD}=0,008)$.

En la Tabla 1 se muestran los resultados medios de la LHS y sus correspondientes ítems, según el sexo y el tipo de EM.

TABLA 1 RESULTADO DE LAS VARIABLES DE LA LHS SEGÚN SEXO Y TIPO DE EM DE LOS USUARIOS

\begin{tabular}{|c|c|c|c|c|c|}
\hline & \multicolumn{2}{|c|}{ Sexo } & \multicolumn{3}{|c|}{ Tipo de EM } \\
\hline & Hombre & Mujer & EMRR & $\begin{array}{l}\text { EM Primaria } \\
\text { Progresiva }\end{array}$ & $\begin{array}{l}\text { EM Secundaria } \\
\text { Progresiva }\end{array}$ \\
\hline & Media (SD) & Media (SD) & Media (SD) & Media (SD) & Media (SD) \\
\hline $\begin{array}{l}\text { Movilidad } \\
\text { (máx. 0,071) }\end{array}$ & $\begin{array}{l}0,045 \\
(0,15)\end{array}$ & $0,008(0,029)$ & $0,019(0,031)$ & $0,020(0,037)$ & 0,038 \\
\hline $\begin{array}{c}\text { Independencia física } \\
\text { (máx. } 0,102)\end{array}$ & $0,059(0,060)$ & $0,022(0,072)$ & $0,046(0,074)$ & $0,018(0,058)$ & $-0,021$ \\
\hline $\begin{array}{l}\text { Ocupación } \\
\text { (máx. 0,099) }\end{array}$ & $-0,018(0,005)$ & $-0,024(0,009)$ & $-0,024(0,009)$ & $-0,019(0,006)$ & $-0,014$ \\
\hline $\begin{array}{l}\text { Integración social } \\
\text { (máx. 0,063) }\end{array}$ & $0,012(0,037)$ & $0,041(0,029)$ & $0,038(0,031)$ & $0,007(0,033)$ & 0,063 \\
\hline $\begin{array}{l}\text { Orientación } \\
\text { (máx. 0,109) }\end{array}$ & $0,009(0,057)$ & $0,032(0,068)$ & $0,020(0,063)$ & $0,014(0,065)$ & 0,109 \\
\hline $\begin{array}{c}\text { Autosuficiencia } \\
\text { económica } \\
\text { (máx. } 0,1 \text { ) }\end{array}$ & $0,049(0,047)$ & $0,048(0,033)$ & $0,051(0,034)$ & $0,044(0,053)$ & 0,033 \\
\hline LHS Total & $0,612(0,612)$ & $\begin{array}{c}0,581 \\
(0,183)\end{array}$ & $0,605(0,177)$ & $0,539(0,194)$ & 0,664 \\
\hline
\end{tabular}




\section{Cuestionario de Salud SF-36}

Después de calcular y analizar los resultados derivados del Cuestionario de Salud SF36, se puede observar cómo el área con mejor porcentaje medio es el de "Bienestar emocional" con un 72,5\%. Por lo contrario, el conjunto de ítems que refiere una peor puntuación es el de "Limitaciones en los roles por la función física" con un 20\%, como se observa en la Tabla 2.

\section{Análisis inferencial}

Con el objetivo de determinar las posibles relaciones existentes entre las variables estudiadas, así como su nexo con el tema principal del trabajo de investigación y poder establecer inferencias entre las mismas, se han aplicado los métodos de análisis no paramétricos, teniendo en cuenta que la muestra se comporta como una distribución no normal.

TABLA 2 PORCENTAJES MEDIOS POR ÁREAS DEL CUESTIONARIO DE SALUD SF-36

\begin{tabular}{ccc}
\hline Área SF-36 & Media (SD) & Mediana \\
\hline Funcionamiento físico & $41,6 \%(28,6)$ & 40 \\
Limitaciones en los roles por la función física & $20 \%(28,7)$ & 0 \\
Limitaciones en los roles por problemas emocionales & $62,2 \%(43,4)$ & 100 \\
Energía/Fatiga & $34 \%(23,8)$ & 30 \\
Bienestar emocional & $72,5 \%(17,7)$ & 76 \\
Funcionamiento social & $64,2 \%(23,1)$ & 62,5 \\
Dolor & $60,8 \%(15,5)$ & 67,5 \\
Salud General & $36,3 \%(15,8)$ & 35 \\
Cambios en la salud & $38,3(24,8)$ & 50 \\
\hline
\end{tabular}

Se ha visualizado que la puntuación total de la LHs guarda relación con los ítems que indican la presencia de limitaciones en los roles por problemas emocionales del cuestionario SF36, ya que una mayor puntuación total en la LHS supone una disminución en las problemáticas relacionadas con la limitación en los roles debido a problemas emocionales $(\mathrm{p}=\mathrm{O}, \mathrm{O} 22)$. Además, la puntuación total de la LHS, posee también relación con los ítems relacionados con el funcionamiento social (concerniente al Cuestionario SF36), obteniendo un mejor funcionamiento social cuando la puntuación de la LHS aumenta $(\mathrm{p}=\mathrm{O}, 014)$.

Además, el ítem "integración social" (que forma parte de la LHS), se encuentra relacionado con la limitación en los roles por problemas emocionales $(p=0,000)$, el bienestar emocional $(\mathrm{p}=\mathrm{O}, \mathrm{O10})$ y el funcionamiento social $(p=0,000)$, estos tres últimos pertenecientes al cuestionario $\mathrm{SF}_{3} 6$. Así, se muestra que los usuarios que otorgan una mayor puntuación a "integración social" manifiestan menos limitaciones en los roles por problemas emocionales, un mejor bienestar emocional y un óptimo funcionamiento social $(\mathrm{p}<\mathrm{O}, \mathrm{O} 1)$.

Se ha identificado también una relación entre los ítems de energía/fatiga y bienestar emocional, con la salud general ( $p=0,031$ y $p=0,036$ respectivamente), obteniendo una peor concepción de la salud general en caso de aportar puntuaciones menores a ítems vinculados con la fatiga y el bienestar emocional, como se muestra en la Figura 2.

Se ha comprobado también que existen diferencias en cuanto a la media de movilidad establecida por la LHS y el sexo de los usuarios, siendo la movilidad mayor en los usuarios varones. Por término medio, los hombres tienen una puntuación 0,036 mayor en el ítem de movilidad de la LHS que las mujeres $(\mathrm{p}=\mathrm{O}, \mathrm{O} 22)$. 


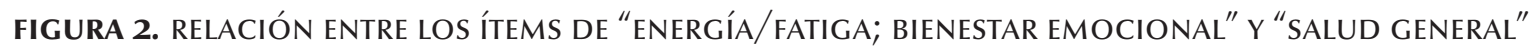

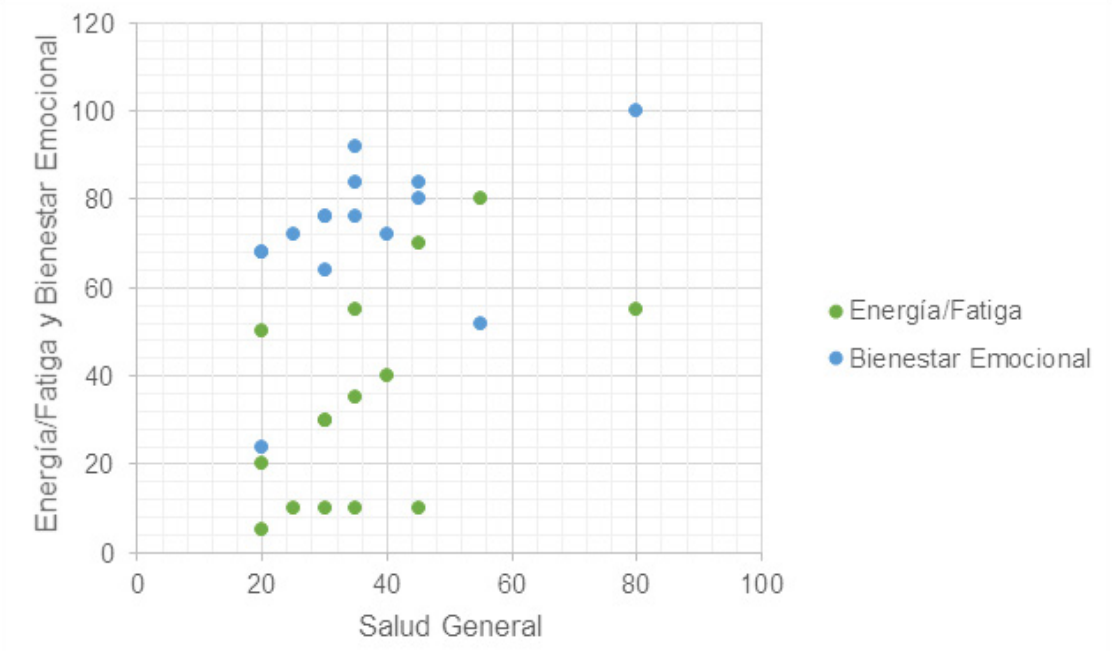

Por su parte, la asistencia al servicio de TO no ha tenido influencia en la valoración de la calidad de vida autopercibida de la persona en el SF36 o de la LHS.

Por último, la LHS ha obtenido un $\alpha$ de Cronbach de $\mathrm{o}, 841$, demostrando una buena consistencia interna en esta herramienta. En lo referente al Cuestionario de Salud $\mathrm{SF}_{3} 6$, ha mostrado un resultado para dicho coeficiente de 0,732 , llegando también al umbral para poder ser calificada como adecuada.

\section{ANÁLISIS DE LOS DATOS CUANTITATIVOS DE LOS PROFESIONALES SOCIO-SANITARIOS}

Según los profesionales encuestados, son minoría los pacientes que reciben tratamiento desde el servicio de TO durante 3 o más días por semana (23,6\%), primando el asistir un día o dos días por semana con un porcentaje del $38,2 \%$ para cada uno de estos últimos.

En otro orden, existe un amplio porcentaje de profesionales $(38,2 \%)$ que no consideran de importancia el incluir en su proceso de evaluación alguna herramienta relacionada con la calidad de vida de los usuarios.

Por otro lado, cuándo se pregunta a los profesionales acerca de cómo valora su trabajo la población afectada y la familia de esta, surgen diferencias relevantes. En una escala del 1 al 5 , dónde $1=$ "muy poco"; y 5="muchísimo", el 61,8\% de los encuestados puntúan con un 4 la valoración por parte de los afectados. Mientras que, en referencia a las familias, los porcentajes más altos se dividen entre la puntuación de 3 y 4, sumando alrededor del $70 \%$.

En la cuestión relativa a los temas tratados durante las sesiones por parte de los profesionales (pudiendo ser escogidos hasta tres temas), destaca el "cómo se encuentra en su día a día" (94,5\%), seguido del "estado emocional" (6o\%). Son pocos los profesionales que tratan los temas relacionados con el "dudas sobre el pronóstico o avance de la enfermedad" $(14,5 \%)$ y "temas laborales" (7,3\%).

Continuando con el análisis de los datos, existen dificultades para clarificar o consensuar cuál sería el abordaje prioritario para dirigir una primera actuación desde la disciplina de TO en los usuarios con EM. La Salud Mental encabeza este apartado con un $41,8 \%$, seguido de la movilidad y rehabilitación física $(33,1 \%)$ y la adaptación del entorno con un 25,1\%. En el lado opuesto, se encuentran las prestaciones orto protésicas, que no ha sido seleccionada por ningún profesional.

En cuanto a la pregunta formulada para conocer si alguno de los profesionales encuestados forma parte de algún equipo de investigación o proyecto relacionado 
con la EM, la gran mayoría contestan negativamente a dicha cuestión, en concreto un $87,3 \%$. Por otro lado, existe un total consenso en lo referido a la necesidad y viabilidad de la intervención domiciliaria desde TO para usuarios con EM, con un $100 \%$ de respuestas afirmativas.

\section{Resultados cualitativos}

A continuación, se exponen, en primer lugar, la detección de las prioridades ocupacionales de los usuarios derivadas de la administración de la copm. Posteriormente, se recogen las principales categorías de significado emergidas tras la lectura y análisis de las entrevistas realizadas a los usuarios, el grupo de discusión y los discursos de los terapeutas ocupacionales que han participado en el cuestionario.

\section{Prioridades ocupacionales a través de la copM}

La сомP ha permitido conocer qué perciben los usuarios con EM como prioritario en su desempeño.

A continuación, en la Tabla 3 se mencionan las diferentes prioridades ocupacionales de los usuarios que posteriormente dieron lugar a las categorías de significado resultantes del análisis del discurso de las entrevistas y grupo focal, en relación a estas.

\section{TABLA 3 PRIORIDADES OCUPACIONALES MÁS COMUNES} DE LOS USUARIOS

\begin{tabular}{ccc}
\hline Actividad & Porcentaje & N \\
\hline Trabajar & $66,7 \%$ & 10 \\
Quedar con amigos & $60 \%$ & 9 \\
Hacer deporte & $46,7 \%$ & 7 \\
Realizar las tareas del hogar & $40 \%$ & 6 \\
Hacer manualidades o coser & $40 \%$ & 6 \\
Ir al cine & $33,3 \%$ & 5 \\
Conducir & $26,7 \%$ & 4 \\
\hline
\end{tabular}

\section{Categorías de significado del fenómeno de estudio}

A continuación, se presentan las categorías de significado obtenidas tras el análisis temático de entrevistas y grupo focal.

\section{El querer y el poder, frente a frente}

La mayor parte de los usuarios muestran cierta frustración ante la imposibilidad de poder realizar todo lo que quieren en su día a día. Existen ciertas actividades que se encuentran comprometidas a causa de la fatiga asociada a la enfermedad. Además, la resiliencia es un factor importante detectado en el discurso de los participantes con relación a alcanzar un adecuado desempeño.

U11: "Yo ahora lo que más noto es que algo que me llevaba dos minutos, ahora me lleva seis, ocho o diez. Tengo que ir mucho más despacio porque no me da. No me da el cuerpo para eso... La fatiga es la peor compañía"

U7: "Simplemente el hecho de hacer la cama o ducharme me agota mucho. Quien no sufre esto y hay mucha gente que no lo entiende, dirán: ; qué exagerada! Pero es que simplemente pues eso, cualquier cosa básica de la vida diaria, que cualquier otra persona no le daría importancia, a mi me cansa muchísimo"

U3: "A lo largo de los años me acostumbré a la realidad... Primero luchas a contracorriente. Luego te das cuenta de que eso no sirve para nada. (...) Es buscar actividad y, por ejemplo, llegar a casa después de hacer la compra y conseguirlo y mandarte mensajes positivos... jBien, lo conseguiste!"

\section{Pérdida del rol de trabajador/a}

El trabajo remunerado ha sido uno de los temas relevantes durante las entrevistas individuales, incluso en mayor grado que las ocupaciones relativas a la socialización o el ocio, dado que la gran mayoría de los afectados se han visto en la obligación de abandonar su trabajo ante la imposibilidad de llevarlo a cabo o compatibilizarlo con el tratamiento rehabilitador adecuado.

Este proceso presenta diversas etapas desde del diagnóstico de la enfermedad pasando por un proceso de 
asimilación desde el momento inicial hasta el desenlace, contemplado como la pérdida del puesto laboral.

Se observa que esta repercute negativamente en la cotidianeidad de los usuarios, con lo que ello implica para su rol social e identidad, viéndose ambos alterados.

U5: "Mi actividad profesional ya no existe y era algo, digamos bastante importante tanto cuantitativa como cualitativamente en mi vida. (...) Yeso... Yo echo mucho de menos trabajar"

U3: "Me gustaría poder volver a trabajar. Yo trabajaba durante los 2 primeros años de la enfermedad, luego ya se acabó. Llevo 16 años sin trabajar y claro... Todo es muy monótono"

\section{Restricciones, barreras y dificultades para la participación en la comunidad}

La presencia de barreras o factores limitantes que restringen la participación de las personas con EM resulta evidente en los discursos de los participantes. El aislamiento y la soledad emergen ante la situación de no poder participar en sus hábitos, rutinas o el no poder estar con las personas que desean, influyendo esto en su participación social y comunitaria.

U3: "Esta enfermedad lo que pasa es, que poco a poco te vas quedando cada vez más sola, porque es así. (...) Yotras veces te vas apartando, yo por lo menos. Porque yo siempre dije que esto es mi problema. No es tu problema. Entonces tendré que llevarlo lo mejor que pueda"

La pérdida del control urinario y de esfínteres, es contemplada como una gran preocupación que condiciona las salidas al exterior y la realización de actividades.

U14: "A mi lo del baño me trae por el camino de la amargura... Es un tema que me preocupa mucho, me paraliza"

\section{Auto-estigma vs estigma social}

La incomprensión por parte de la ciudadanía ante la EM es notable según los pacientes, haciendo referencias habituales en los discursos. Los productos de apoyo como muletas, bastones o scooters, han sido identificados por los usuarios como atributos negativos.
U1: "La gente tiene diferentes perspectivas por ejemplo de un bastón a una muleta. Si voy con la muleta parece que tuviste una lesión en la rodilla o que te caíste haciendo deporte... Pero ¿con el bastón? Dicen jo pobre, que le pasa..."

El concepto de auto-estigma brota en los discursos de los informantes, hablando de este cuando es la propia persona afectada la que reproduce esos prejuicios y la discriminación hacia sí misma o hacia su colectivo. La importancia del entorno es fundamental en este aspecto, ya que los usuarios manifiestan no ser impermeables a lo que les rodea.

U2: "Estoy limitada, ¿no? Yo no puedo irme por ahía caminar o a hacer cosas que hacen los demás... Taly como camino además, no puedo ir por ahí... Van a pensar que estoy no sé... Y con razón"

\section{Los servicios socio-sanitarios: a debate}

Son numerosas las referencias a la ausencia de unos servicios continuos de tratamiento rehabilitador y otros servicios de salud necesarios en el abordaje integral de la EM, tanto por parte de las personas afectadas como por parte de los profesionales participantes. Estos destacan aspectos relacionados con las barreras presentes en los recursos que condicionan el desarrollo de la intervención con esta población, la promoción del trabajo transdisciplinar y el abogar por una intervención domiciliaria desde TO en el abordaje de la EM.

U3: "La seguridad social nunca me dio rehabilitación. Me la tuve que buscar yo y pagarla de mi bolsillo. No te la dan así gratuita. Habría que pelear... Ya que tanto te lo recomiendan..."

P1: "La principal limitación, a mi modo de ver, de la intervención desde TO es el tiempo: dada la escasez de recursos y la elevada cantidad de personas potencialmente usuarias, las sesiones de intervención son, a menudo, mucho más cortas de las que sería ideales para la consecución de objetivos"

P8: "Sería importante mejorar la coordinación interprofesional para aumentar la participación de los usuarios en tratamientos integrales de rehabilitación funcional (muchos se centran únicamente en el tratamiento fisioterápico) 


\section{Discusión}

El objetivo principal del presente estudio ha sido explorar y conocer cómo las personas con EM (en este caso, de la Comunidad Autónoma de Galicia) perciben su estado de salud y calidad de vida con relación a su participación y desempeño en lo cotidiano, tanto individual como colectivamente. Con ello, se ha intentado dar voz más allá de una etiqueta diagnóstica, destacando el auto-estigma y la autopercepción de la competencia ocupacional relacionada con la enfermedad, aspectos un tanto olvidados en esta población pero que son de gran importancia para los propios usuarios. Del mismo modo, se ha procurado conocer y compartir de la mano de los profesionales de TO su percepción acerca de la EM, la población que la posee y la intervención terapéutica desarrollada. Dichos objetivos generales se han alcanzado, determinando las necesidades ocupacionales de las personas con EM, conociendo y dando significado a los diversos fenómenos que interfieren en la calidad de vida y bienestar en términos ocupacionales de esta población.

El abordaje del análisis de la calidad de vida y percepción del estado de salud en personas con EM permite conocer una perspectiva global de las mismas destacando el cuestionario de salud SF-36 como uno de los más utilizados en el ámbito de la EM (Terré \& Orient, 2007). Existen diversas herramientas estandarizadas que permiten profundizar en los niveles de calidad de vida de personas con EM. Sin embargo, y sorprendentemente, casi un $40 \%$ de los profesionales de TO que han participado no considera de importancia utilizar alguna de dichas herramientas para conocer la calidad de vida de las personas afectadas. Este dato resulta impactante ante la literatura científica existente, en la que se aprecia una alta utilización de instrumentos estandarizados: SF-36 o Multiple Sclerosis Quality of Life- 54 entre ellas (Newland, Lunsford \& Flach, 2017; Hadgkiss, Jelinek, Weiland, Rumbold, Mackinlay \& Gutbrod, 2013; Camfield \& Skevington, 20o8; Pozzilli, Brunetti, Amicosante, Gasperini, Ristori, Palmisano \& Battaglia, 2005)

En cuanto a los resultados obtenidos tras la aplicación de la SF-36, en el presente estudio, el ítem con mejor puntuación ha sido el de "bienestar emocional". Con este hallazgo puede establecerse un paralelismo con el dato arrojado por parte de los profesionales, que afirmaron con $41,8 \%$ que la intervención en Salud Mental debía ser el primer abordaje realizado desde TO en esta población. En consonancia con esa respuesta, existen estudios que destacan que es sumamente importante considerar la influencia de la situación psicoemocional en el transcurso de la EM (Strober, 2018; Wilski, Kocur, Górny, Koper, Nadolska \& Chmielewski, 2019). Además, se percibe la figura del terapeuta ocupacional como imprescindible para explorar y analizar cómo los problemas derivados de la salud mental afectan a la vida diaria de las personas y a su calidad de vida (Mesa, Anderson, Askey-Jones, Gray, \& Silber, 2012). Entre los diversos factores predisponentes para la aparición de un proceso depresivo en personas con EM, se encuentran la ausencia o falta de apoyo social, altos niveles de auto-estigma y la propia discapacidad física (Maurino, Martínez-Ginés, García-Domínguez, Solar, CarcelénGadea, Ares-Luque, Ballabriga, Navarro-Cantó, Medrano \& Honan, 2020). Esto se ve reforzado con los resultados del presente estudio, derivados de los diferentes discursos recogidos en los resultados acerca del mencionado auto-estigma o ante el escaso apoyo social. Del mismo modo se indica que el hecho de abordar con los usuarios ciertos temas acerca de cómo influye la EM en su calidad de vida, puede ofrecer información muy valiosa sobre su salud, siendo esencial para llevar a cabo una práctica centrada en la persona (Pérez de HerediaTorres, Huertas-Hoyas, Sánchez-Camarero, MáximoBocanegra, Alegre-Ayala, Sánchez-Herrera-Baeza et al., 2020). Además, la disciplina de TO posee un papel muy importante en la atención a los usuarios con EM y sus posibles condicionantes de Salud Mental. Para ello, recomiendan, además del uso de herramientas validadas e intervenciones basadas en la evidencia desde TO, su participación en proyectos de investigación de la EM y Salud Mental (Mesa et al., 2012). A través de ello, se conseguiría ampliar el conocimiento existente de la EM y las respuestas asociadas a la salud mental de las personas sobre el desempeño ocupacional y su participación en la vida diaria, roles sociales y calidad de vida.

En una revisión acerca del rol investigador del terapeuta ocupacional en la EM, destacan la necesidad de buscar la eficacia en otro tipo de intervenciones desde TO (en consonancia con los discursos de los profesionales participantes) como las adaptaciones del entorno y la reeducación de las actividades de la vida diaria (López, Martínez, \& Martínez, 2018). Por el contrario, también destacan la importancia de la función del profesional en la prescripción y entrenamiento de productos de apoyo u ortroprotésicos, algo que ninguno de los terapeutas ocupacionales encuestados ha decidido marcar 
como prioritario en la pregunta relacionada con el primer abordaje de la EM desde la disciplina. Es un dato impactante, dada la conocida necesidad con relación a los dispositivos de apoyo u otras prestaciones ortoprotésicas de la población con EM.

Además, el 100\% de los terapeutas ocupacionales que respondieron el cuestionario, mostraban su conformidad ante la viabilidad y la necesidad de la intervención domiciliaria de TO en usuarios con EM. Otros trabajos (ya desde inicios de los 2000) reflejan en sus resultados que tras comparar la intervención hospitalaria y la domiciliaria, los usuarios del grupo de estudio, muestra un aumento en las puntuaciones de la SF-36 frente a los que han recibido una intervención clásica (Williams, Low-Choy \& Brauer, 2021; Kezele, Babić \& Štimac, 2019; Pozzilli et al., 2005). Desde la perspectiva de la TO, se conocen los beneficios que aporta una intervención en el entorno natural y propio del usuario. Sin embargo, es algo que todavía sigue sin incluirse completamente en los protocolos de intervención y asistencia del sistema público de salud.

Las AVDB y AVDI han sido algunas de las actividades más mencionadas por los usuarios tanto en los discursos de la entrevista como en la COPM. Ciertas actividades como hacer la cama, ducharse, realizar el aseo personal u ordenar, se complican con la presencia de la EM y, sobretodo, de su principal síntoma: la fatiga. Estos resultados coinciden con los arrojados por otros estudios donde las actividades pertenecientes al área del autocuidado fueron detectadas como las más importantes con un $42,3 \%$ de las menciones (Carolina, Rodrigues De Oliveira, Sousa De Andrade, Gontijo, \& Barroso, 2013) o alcanzando un $57,4 \%$ en un reciente estudio del año 2020 (Pérez de Heredia-Torres et al., 2020). En consonancia, otro estudio publicado en el año 2018 refleja que el $100 \%$ de la muestra de estudio $(n=200)$ identifica en las actividades de autocuidado un menor grado de satisfacción sobre su desempeño (Abdullah, Badr, \& Manee, 2018). Además, y en relación a la fatiga, los usuarios la consideran uno de los factores más limitantes en su vida, al igual que los participantes del estudio publicado en la revista Disability and Rehabilitation. Estos han descrito que la fatiga es también para ellos una de las principales barreras para participar en las actividades de la vida diaria, interfiriendo en la adaptación a los desafíos que implica la EM (Silverman, Verrall, Alschuler, Smith, \& Ehde, 2017). Ante esta situación, se puede hablar de una posible ruptura con las AVD's de la población con EM, como relataban la mayor parte de los participantes, dejando de realizar algunas actividades y/o necesitando un mayor tiempo de desarroIlo. Con ello, se establece lo que Whiteford denomina como "disrupción ocupacional" y que define como "el estado temporal o transitorio que ocurre cuando un patrón normal de compromiso ocupacional se ve interrumpido debido a eventos significativos de la vida". Añade que se trata de un estado temporal el cual, con las condiciones de apoyo adecuadas, se resuelve. Aunque a lo largo del trabajo se hayan realizado menciones acerca del carácter progresivo de la enfermedad, el hecho de aprender a convivir con ella y conocer técnicas para suplir o mejorar el desempeño, lleva a la resolución de ese estado de conflicto (Whiteford, 2000).

Al igual que en las AVDB y AVDI, la actividad laboral también ha adquirido un valor muy importante en la rutina diaria de los participantes tras la llegada de la EM, donde la fatiga y la problemática a nivel cognitivo juegan un papel fundamental (Koblet, Langdon \& Jönsson, 2018). Otros autores relatan que la pérdida del puesto de trabajo es una de las mayores preocupaciones de los usuarios con EM (García-Domínguez, Maurino, Martínez-Ginés, Carmona, Caminero \& Medrano, 2019; Cores, Vanotti, Burin, Politis, \& Villa, 2014). El cambio del rol de trabajador puede generar una disminución de la autoestima y gran frustración. Es por ello que también se producen alteraciones en la salud y en la calidad de vida de los usuarios tras la pérdida de dicha actividad productiva (Vidal \& Abal, 2021; Vijayasingham \& Mairami, 2018). Además, en la gran mayoría de los casos, la autoestima se encuentra relacionada con los logros laborales, sufriendo una desestructuración en los objetivos de vida de los usuarios, teniendo que ser modificados o adaptados.

Diferentes autores, tras analizar las prioridades ocupacionales de los usuarios a través de la COPM, destacaron que el trabajo fue mencionado por el $26,3 \%$ de los usuarios (Carolina et al., 2013). Los datos arrojados concuerdan con esta investigación, en el que el $66,7 \%$ señala el empleo o puesto de trabajo como una necesidad en su vida cotidiana. De hecho, diez de los quince usuarios participantes en el presente estudio han identificado el trabajo como una prioridad en su día a día, ocupación que les gustaría recuperar.

El desarrollo de un trabajo de investigación de estas características donde la persona es el eje central junto 
a su percepción en relación a su cotidianidad (y de un colectivo o comunidad al que pertenece) resulta muy enriquecedor para una disciplina como la TO, donde se mantiene una visión holística de la persona siendo ésta valorada como tal en el desarrollo y selección de sus ocupaciones significativas en la sociedad o comunidad, participando de manera libre en las mismas, siendo la base de una sociedad justa e inclusiva, como sostiene el "Position Statement: Occupational Therapy and Human Rights" (World Federation of Occupational Therapists, 2019). Además, el hecho de incluir en el proceso metodológico la aplicación de herramientas estandarizadas para conocer los niveles de independencia, calidad de vida autopercibida o prioridades ocupacionales, posibilita el enriquecimiento de los resultados.

\section{CONCLUSIONES}

Tras la realización del presente estudio y un análisis exhaustivo de sus resultados, se puede concluir que:

El estigma hacia la enfermedad define un patrón negativo en la calidad de vida de la población de estudio. Además, la fatiga condiciona su participación en actividades (tanto básicas como instrumentales), sobretodo afectando al rol de trabajador/a que proporciona gran significado a la identidad de la persona.

En definitiva, se puede establecer que existe una necesidad (demandada tanto por parte de afectados como de profesionales) de cambio en el enfoque de la atención a las personas con EM, contemplando factores centrales para la cotidianeidad desde un prisma biopsicosocial.

\section{Agradecimientos}

Agradecer a FEGEREC, a su directora y a sus profesionales, con especial mención a Candela Presedo, el permitirnos realizar el estudio en su entidad. A todos los y las participantes por acceder a realizarlo.

\section{RefERENCIAS BibLIOGRÁFICAS}

Abdullah, E. J., Badr, H. E., \& Manee, F. (2018). Ms people's performance and satisfaction with daily occupations: Implications for occupational therapy. OTJR Occupation, Participation and Health, 38(1), 28-37. https://doi.org/10.1177/1539449217719867
Arbinaga, F. (2003). Aspectos emocionales y calidad de vida en pacientes con enfermedades desmielinizantes: El caso de la EM. Anales de Psicología, 19, 65-74.

Bermejo, F., Porta, J., Díaz J. \& Martínez, P. (2008). Más de cien escalas en neurología (2a). Madrid, España: Aula Médica.

Camfield, L., \& Skevington, S. M. (2008). On subjective well-being and quality of life. J Health Psychol, 24, 2023-2034.

Cano, M. I. (2015). Atención e intervención social en personas con esclerosis múltiple. Una aproximación a la realidad de la práctica profesional. Trabajo Social Hoy, 76, 97-110. https://doi. org/10.12960/TSH.2015.0017.TRABAJO

Carolina, A., Rodrigues De Oliveira, F., Sousa De Andrade, V., Gontijo, D. T., \& Barroso, S. M. (2013). Caracterização e queixas relacionadas ao desempenho ocupacional: considerações de indivíduos com esclerose múltipla/Characterization and complains related to occupational performance: considerations of people with multiple sclerosis. Rev. Ter. Ocup. Univ. São Paulo, 24(2), 112-120. https://doi.org/10.11606/issn.2238-6149.v24i2p112-120

Cores, E.V., Vanotti, S., Burin, D. I., Politis, D. G., \& Villa, A. (2014). Factores asociados con la situación laboral de pacientes con esclerosis múltiple. Rev Neurol, 58(4), 175-183.

Esclerosis Múltiple España. (2019) Día Mundial de la Esclerosis Múltiple 2019: cifras, reivindicaciones y actividades. Retrieved March 31, 2021 from https://www.esclerosismultiple.com/esclerosismultiple/que-es/

Federación Gallega de Enfermedades Raras y Crónicas (2018). Misión, visión y valores. Retrieved February 19, 2019, from http://www. fegerec.es/FEGEREC

Federación Gallega de Esclerosis Múltiple. (2018). Calidad de vida y cifras de Esclerosis Múltiple. Retrieved from https://esclerosismultiplegalicia.org/calidad_vida_cifras_esclerosis_multiple_es.html

García-Cano, B., \& García-Cano, F. (2017). Epidemiología de la Esclerosis Múltiple: revisión bibliográfica. Hygia de Enfermería, 94, 24-27. Retrieved from www.colegioenfermeriasevilla.es

García, A., Ara, J. R., Fernández, O., Landete, L., Moral, E., \& RodríguezAntigüedad, A. (2017). Consenso para el tratamiento de la esclerosis múltiple 2016. Sociedad Española de Neurología. Neurología, 32(2), 113-119. https://doi.org/10.1016/j.nrl.2016.02.026

García, F., \& Musitu, G. (2014). AF5. Autoconcepto Forma 5. Madrid, España: TEA.

García-Domínguez, J., Maurino, J., Martínez-Ginés, M., Carmona, O., Caminero, A., \& Medrano, N. (2019). Economic burden of multiple sclerosis in a population with low physical disability. BMC Public Health, 19(1).

Hadgkiss, E. J., Jelinek, G. A., Weiland, T. J., Rumbold, G., Mackinlay, C. A., Gutbrod, S., \& et al. (2013). Health-related quality of life outcomes at 1 and 5 years after a residential retreat promoting lifestyle modification for people with multiple sclerosis. Neurol Sci, 34, 187-195. 
Harwood, R. H., \& Ebrahim, S. (1995). Manual of the London Handicap Scale. Nottingham: Department of Health Care of the Elderly.

Harwood, R. H., Rogers, A., Dickinson, E., \& Ebrahim, S. (1994). The London Handicap Scale; a new outcome measure for chronic disease. Qual Health Care, 3, 11-16.

Hernandez, R., Fernández, C., \& Baptista, P. (2010). Metodología de la investigación. (I. E. SA, Ed.) (5ª). México.

Kezele, T. G., Babić, M., \& Štimac, D. (2019). Exploring the feasibility of a mild and short 4-week combined upper limb and breathing exercise program as a possible home base program to decrease fatigue and improve quality of life in ambulatory and non-ambulatory multiple sclerosis individuals. Neurol Sci, 40(4), 733-743.

Kielhofner, G. (2006). Fundamentos Conceptuales de la Terapia Ocupacional ( $\left.3^{\mathrm{a}}\right)$. Panamericana.

Kielhofner, G. (2008). Model of Human Occupation: theory and application. (M. D. Baltimore, Ed.). Lippincott Williams \& Wilkins.

Kobelt, G., Langdon, D., \& Jönsson, L. (2018). The effect of self-assessed fatigue and subjective cognitive impairment on work capacity: The case of multiple sclerosis. Multiple Sclerosis Journal, 25(5), 740-749.

Koch-Henriksen, N., \& Sørensen, P. S. (2010). The changing demographic pattern of multiple sclerosis epidemiology. The Lancet Neurology, 9(5), 520-532. https://doi.org/10.1016/S1474-4422(10)70064-8

Kos, D., Duportail, M., Meirte, J., Meeus, M., D'hooghe, M. B., Nagels, G., \& Nus, J. (2016). The effectiveness of a self-management occupational therapy intervention on activity performance in individuals with multiple sclerosis-related fatigue: a randomized-controlled triall. International Journal of Rehabilitation Research, 39(3), 255-262.

Kurtzke, J. F. (2009). A reassessment of the distribution of multiple sclerosis: Part One. Acta Neurol Scand, 57(2), 110-136.

López, N., Martínez, M., \& M, Ma. (2018). El rol investigador del terapeuta ocupacional en la esclerosis múltiple. Revista Argentina de Terapia Ocupacional, 4(1), 47-51.

Maurino, J., Martínez-Ginéz, M. L., García-Domínguez, J. M., Solar, M. D., Carcelén-Gadea, M., Ares-Luque, A., \& Honan, C. A. (2020). Workplace difficulties, health-related quality of life, and perception of stigma from the perspective of patients with Multiple Sclerosis. Mult Scler Relat Disord, 41.

Mesa, A., Anderson, K. H., Askey-Jones, S., Gray, R., \& Silber, E. (2012). The Mental Health Needs of Individuals Living With Multiple Sclerosis: Implications for Occupational Therapy Practice and Research. Mental Health Spec Interest Sect Q, 35(2), 1-4. Retrieved from http://www.ncbi.nlm.nih.gov/pubmed/25309118

Miric, M., Álvaro, J. L., González, R., \& Torres, A. R. R. (2018). Microsociología del estigma: aportes de Erving Goffman a la conceptualización psicosociológica del estigma social. Psicologia e Saber Social, 6(2), 172-185. https://doi.org/10.12957/psi.saber. soc. 2017.33552
Newland, P. K., Lunsford, V., \& Flach, A. (2017). The interaction of fatigue, physical activity, and health-related quality of life in adults with multiple sclerosis (MS) and cardiovascular disease (CVD). Appl Nurs Res, 33, 49-53.

Novartis. (2018). Estudio sobre la Percepción de las personas con EM sobre su calidad de vida Informe de resultados. (n.d.). Retrieved from https://www.novartis.es/sites/www.novartis.es/files/ Informe ME Interesa.PDF

Pérez-Carmona, N., Fernández-Jover, E., \& Sempere, Á. P. (2019). Epidemiology of multiple sclerosis in Spain. Revista de Neurologia, 69(1), 32-38. https://doi.org/10.33588/rn.6901.2018477

Pozzilli, C., Brunetti, M., Amicosante, A. M. V, Gasperini, C., Ristori, G., Palmisano, L., \& Battaglia, M. (2005). Home based management in multiple sclerosis: Results of a randomised controlled trial. Journal of Neurology Neurosurgery and Psychiatry, 73(3), 250-255. https://doi.org/10.1136/jnnp.73.3.250

Prieto-González, J. M. (2000). Escalas de valoracion funcional en la esclerosis multiple. Rev Neurol, 30(12), 1246-1252.

Silverman, A. M., Verrall, A. M., Alschuler, K. N., Smith, A. E., \& Ehde, D. M. (2017). Bouncing back again, and again: a qualitative study of resilience in people with multiple sclerosis. Disability and Rehabilitation, 39(1), 14-22. https://doi.org/10.3109/0963 8288.2016.1138556

Strober, L. B. (2018). Quality of life and psychological well-being in the early stages of multiple sclerosis (MS): importance of adopting a biopsychosocial model. Disabil Health J, 11, 555-561.

Terré, R., \& Orient, F. (2007). Tratamiento rehabilitador en la esclerosis múltiple. Rev Neurol, 44(7), 426-431. Retrieved from http:// www.esclerosismultipleeuskadi.org/que-es-la-em/aspectosgenerales-de-la-em/

Vaismoradi, M., Turunen, H., \& Bondas, T. (2013). Content analysis and thematic analysis: Implications for conducting a qualitative descriptive study. Nurs Heal Sci, 15, 398-405.

Vidal, V., \& Abal, P. (2021). Alimentación, descanso y sueño: repercusiones en el desempeño ocupacional de las personas con esclerosis múltiple. Npunto, IV, 165-171.

Vijayasingham, L., \& Mairami, F. F. (2018). Employment of patients with multiple sclerosis: the influence of psychosocial-structural coping and context. Degener Neurol Neuromuscul Dis, 26(8), 15-24.

Whiteford, G. (2000). Occupational Deprivation: Global Challenge in the New Millennium. BR J Occup Ther, 63(5), 200-204.

Williams, K. L., Low-Chow, N. L., \& Brauer, S. (2020). Center-Based Group and Home-Based Individual Exercise Programs Have Similar Impacts on Gait and Balance in People With Multiple Sclerosis: A Randomized Trial. American Academy of Physical Medicine and Rehabilitation, 13(1), 9-18.

Wilski, M., Kocur, P., Górny, M., Koper, M., Nadolska, A., Chmielewski, B., \& et al. (2019). Perception of Multiple Sclerosis Impact and Treatment Efficacy Beliefs: Mediating Effect of Patient's Illness and Self-Appraisals. J Pain Symptom Manage, 58, 437-444. 
World Federation of Occupational Therapists. (2019). Position statement, Occupational Therapy and Human Rights (revised), 1-2. Retrieved from https://cutt.ly/aflM8cL

Yu, C. H., \& Mathiowetz, V. (2014a). Systematic review of occupacional therapy related interventions for people with multiple sclerosis: Part 2. Impairment. American Journal Of Occupational Therapy, 68(1), 33-38.

Yu, C. H., \& Mathiowetz, V. (2014b). Systematic review of occupational therapy related interventions for people with multiple sclerosis: Part 1. Activity and participation. American Journal OfOccupational Therapy, 68(1), 27-32. 